International Journal of Pure and Applied Mathematics

Volume 102 No. 4 2015, 653-669

ISSN: 1311-8080 (printed version); ISSN: 1314-3395 (on-line version)

url: http://www.ijpam.eu

doi: http://dx.doi.org/10.12732/ijpam.v102i4.6

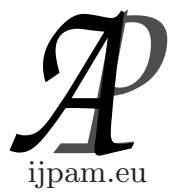

\title{
A CONTROL THEORY APPROACH AIMED AT SUSTAINABLE CONSERVATION OF SINGLE SPECIES UNDER HUMAN INTERVENTION
}

\author{
Erica Cruz-Rivera ${ }^{1}$, Olga Vasilieva ${ }^{2} \S$ \\ ${ }^{1,2}$ Department of Mathematics \\ Universidad del Valle \\ Calle 13 No. 100-000 Cali, COLOMBIA
}

\begin{abstract}
In this work, we consider an infinite-horizon bioeconomic growth model that links the biological evolution of a single species with the capital accumulation dynamics. The biological species has no harvesting value and we presume that there is a social planner acting in the economy whose decision preferences favor the long-term conservation of the species.
\end{abstract}

AMS Subject Classification: 92D25, 49K15, 34H05

Key Words: logistic model, optimal control, maximum principle, nonharvesting utility, optimal-choice policies

\section{Introduction}

In recent decades, there has been noted an increasing interest in research on relation and interaction between economic growth, sustainable development, and natural environment conservation.

There are many wildlife species with no harvest value that are currently in threat by negative side-effects of human activities (urbanization, pollution, habitat loss, etc.). To study the evolution of such species, a non-harvesting variant of dynamic model for one particular species was initially proposed in

Received: January 18, 2015

(C) 2015 Academic Publications, Ltd.

$\S$ Correspondence author url: www.acadpubl.eu 
[1]. This model was formulated in terms of optimal control using as a control variable an index of the quantity of land resources which are vital for the species survival. However, this non-harvesting model did not explicitly include the negative impact that human activities and aggregated production may have on the natural evolution of species population.

Other scholars (see e.g. $[2,4,6,7,10]$ ) had analyzed how the aggregated production and human actions (both negative and positive) may alter the stability properties of the natural dynamic of non-commercial biological species.

We presume that there is a social planner acting in the economy who chooses the levels of consumption and investments in defensive expenditures so as to maximize the present discounted value of total utility subject to the physical capital accumulation dynamics and the amended ecological dynamics. The utility function considered in this paper favors both the societal consumption and the species conservation ${ }^{1}$, although the biological species might be threatened by local extinction.

\section{A Bioeconomic Model with Logistic Growth}

Let us suppose that a single-species population $x(t)$ obeys the evolutionary biological dynamics given by logistic equation under the course of nature without human intervention. Eventually, the biological dynamics can be affected by side-effects of human activity such as aggregated production, pollution, urbanization, etc. By introducing a simple growth model, the species biological evolution can be linked to a capital accumulation dynamics. For the sake of simplicity, we assume that there is a single good which is produced by the capital $k(t)$ itself (see $[4,6,7,10]$ for more details regarding the model's description). As a result we obtain a bioeconomic ODE system

$$
\begin{cases}\frac{d x}{d t}=r x(t)\left(1-\frac{x(t)}{K}\right)-\epsilon k^{\alpha}(t)+\sigma d^{\mu}(t), & x(0)=x_{0}, \\ \frac{d k}{d t}=k^{\alpha}(t)-c(t)-d(t), & k(0)=k_{0},\end{cases}
$$

whose entries are defined in the Table 1 . Here $x_{0}>0$ and $k_{0}>0$ are initial values of the species population and the capital, respectively. It should be noted that parameter $\mu \in(0,1)$ is introduced in order to emphasize that the positive effect of defensive investment on the specie evolution is not directly

\footnotetext{
${ }^{1}$ The same utility function was treated in [2] and [4].
} 


\begin{tabular}{lll}
\hline Notation & Description & Role \\
\hline$x(t)>0$ & species population at the moment $t$ & state \\
$k(t)>0$ & cumulative capital at the moment $t$ & state \\
$c(t)>0$ & consumption at the moment $t$ & control \\
$d(t) \geq 0$ & defensive expenditures at the moment $t$ & control \\
$r>0$ & intrinsic growth rate of the species population & constant \\
$K \gg 0$ & carrying capacity of the species population & constant \\
$0<\alpha<1$ & parameter of the Cobb-Douglas production function $k^{\alpha}$ & constant \\
$0<\mu<1$ & parameter related to defensive expenditures & constant \\
$\epsilon>0$ & measure of the negative impact of aggregated production $k^{\alpha}$ & constant \\
$\sigma>0$ & measure of the positive impact of defensive expenditures $d^{\mu}$ & constant \\
\hline
\end{tabular}

Table 1: Problem entries

proportional to population growth; in other words, extra-spending on species conservation (increase in $d(t))$ may decrease the positivity of such impact on the evolution of $x(t)$ due to the carrying capacity limitations of the environment.

The dynamics of the bioeconomic system (1) will not be as stable as biological dynamics given by traditional logistic equation and $\underline{x}^{*}=0, \bar{x}^{*}=K$ will not be its fixed points. In terms of stationary structures, the fixed points of (1) will naturally depend on the parameters $c, d$ and can be expressed as

$$
x_{ \pm}^{*}=\frac{K}{2}\left(1 \pm \sqrt{1+\frac{4}{r K}\left[\sigma d^{\mu}-\epsilon(c+d)\right]}\right), \quad k^{*}=(c+d)^{1 / \alpha} .
$$

Note that $x_{ \pm}^{*}$ will be real numbers only if

$$
\sigma d^{\mu}-\epsilon(c+d)+\frac{r K}{4} \geq 0
$$

Fulfillment of condition (3) will also guarantee that $0 \leq x_{-}^{*} \leq x_{+}^{*} \leq K$ and that $x_{-}^{*}+x_{+}^{*}=K$ due to symmetry of the first relation in (2) and independently of the parameters choice. Thus, we have obtained a natural constraint for the parameters $c, d$ in the form (3) that indicates so-called "confidence region" provided that $c>0$ and $d \geq 0$ for all $t$.

It is easy to prove (see [6]) that $\left(x_{+}^{*}, k^{*}\right)$ is saddle point and $\left(x_{-}^{*}, k^{*}\right)$ is nodal repeller. Thus, instead of stable equilibrium natural for logistic equation, we now have (in the best case) only a stable manifold of the saddle point $\left(x_{+}^{*}, k^{*}\right)$.

Now, if $\epsilon>0$ is set free while other parameters of the model (including $c$ and $d$ ) remain constant, then $x_{ \pm}^{*}=x_{ \pm}^{*}(\epsilon)$ will be functions of $\epsilon$ and we can determine particular values $\hat{\epsilon}$ and $\epsilon_{0}$ such that

$$
\left.\hat{\epsilon}=\frac{\sigma d^{\mu}}{c+d}: \quad x_{-}^{*}(\hat{\epsilon})=0, \quad x_{+}^{*}(\hat{\epsilon})=K \quad \text { (logistic case }\right)
$$




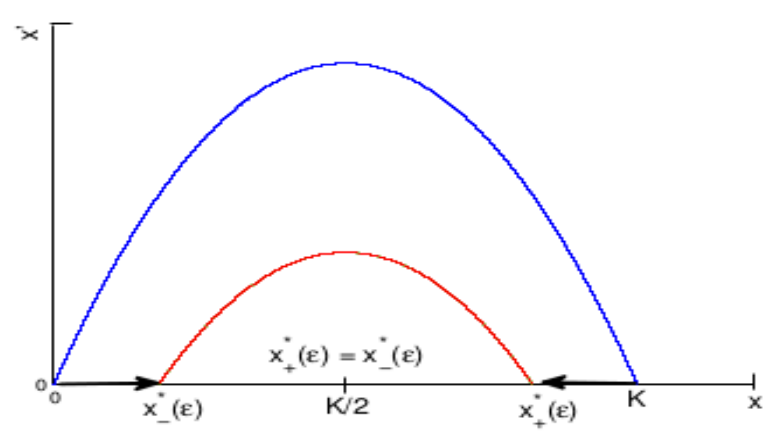

Figure 1: Displacements of the fixed points $x_{-}^{*}(\epsilon)$ and $x_{+}^{*}(\epsilon)$ when $\epsilon$ varies between $\hat{\epsilon}$ and $\epsilon_{0}$.

$$
\epsilon_{0}=\frac{4 \sigma d^{\mu}+r K}{4(c+d)}: \quad x_{-}^{*}\left(\epsilon_{0}\right)=x_{+}^{*}\left(\epsilon_{0}\right)=\frac{K}{2} \quad \text { (degenerate case) }
$$

From the ecological point of view, $\hat{\epsilon}>0$ can be called opportune reference value since it characterizes an "ideal situation", that is, both positive and negative effects of aggregated production do not alter the natural equilibrium of the dynamic system. On the other hand, $\epsilon_{0}$ can be called critical reference value since it characterizes an "unhealthy situation" when the equilibrium of the system is unreachable in infinite time $t$. The latter also implies that Jacobian matrix evaluated in $x^{*}=K / 2$ becomes degenerate and has zero as an eigenvalue:

$$
\operatorname{det} J\left(\frac{K}{2}, k, c, d\right)=\operatorname{det}\left[\begin{array}{rr}
0 & -\epsilon \alpha k^{\alpha-1} \\
0 & \alpha k^{\alpha-1}
\end{array}\right]=0 .
$$

Thus, for $\epsilon \neq \hat{\epsilon}$ and $\epsilon \neq \epsilon_{0}$ we have $0<x_{-}^{*}(\epsilon)<x_{+}^{*}(\epsilon)<K$ and $x_{-}^{*}(\epsilon)+x_{+}^{*}(\epsilon)=$ $K$; the latter is illustrated by phase diagram in $\left(x^{\prime}, x\right)$-plane given in the Figure 1. Further on, we shall refer to $x_{-}^{*}(\epsilon)$ as minimum-abundance fixed point and to $x_{+}^{*}(\epsilon)$ as maximum-abundance fixed point.

In general, both reference values $\hat{\epsilon}$ and $\epsilon_{0}$ can be determined outside of the model in accordance with other parameters, and then provide us some useful information regarding to actual ecological situation described by the model (1). Naturally, if the value of $\epsilon$ given in the model (1) is close to $\hat{\epsilon}$, then we say that ecological situation is "good"; otherwise, if $\epsilon$ is close to $\epsilon_{0}$, the situation is "bad".

However, there is still an open question on how $c$ and $d$ are to be chosen in order to calculate $\hat{\epsilon}$ and $\epsilon_{0}$ according to the formulae (4)-(5). To answer that question, we opt for an optimal choice of $c$ and $d$ by introducing a maximumutility criterion and then arriving to an optimal control problem. 


\section{Optimal Control Problem and Optimality System}

Suppose that there is a social planner acting in the economy whose task is to maximize the present discounted value of the utility function $U$ of the society over infinite horizon by choosing appropriately the values of two parameters $c$ and $d$. The society's welfare depends, in each instant $t$, on the consumption $c$ and abundance of the specie $x$, while $d$ represents investments for saving $x$ from (local) extinction. It should be emphasized that $U(x, c)$ has purely nonharvesting value; in other words, the society does not derive any benefit from direct consumption of the species. By defining $c=c(t)>0$ and $d=d(t) \geq 0$ as real-valued piecewise continuous functions, we can now formulate the following optimal control problem:

$$
\int_{t_{0}}^{\infty} U(x, c) e^{-\rho t} d t \rightarrow \max
$$

subject to dynamic system (1). Here the constant parameter $\rho>r>0$ indicates the discount rate which is strictly higher than reproduction rate of biological species $r$.

Remark 1. According to [5], low growth rates of species population relative to discount rate (that is, $\rho>r>0$ ) will lead to stock extermination in common-property models with harvesting. Alternatively, [1] have proved that relation $\rho>r>0$ is admissible for non-harvesting models in the sense that there exists an optimal strategy helping to increase the population of the species.

The definition of utility function $U(x, c)$ must clearly reflect the priorities of decision-making. In [6] we have considered a realistic situation expressed by a utility function which was not adverse to local extinction of the species (i.e., $x=0$ was an acceptable solution). Here we propose a more "ecologically friendly" variant and define a purely logarithmic function. More precisely, we can borrow the exact form of $U(x, c)$ from the paper [2] and set

$$
U(x, c)=\eta \ln x+\nu \ln c,
$$

where $\nu, \eta>0$ are some assigned weight parameters. Function (8) expresses that the presence of the species is crucial for social planning since this species plays a key role in the local ecosystem. Mathematically, $x \approx 0$ will not be acceptable as optimal solution and for $x \rightarrow 0^{+}$there will an infinite loss of 
utility. Additionally, function (8) may reflect, by setting $\eta>\nu$, that species conservation is more important than consumption $c(t)$.

In summary, functional form of $U(x, c)$ given by (8) expresses the preferences of decision-making that are strictly adverse to (local) extinction of the species and thus foster its conservation.

The current-value Hamiltonian of the optimal control problem with infinite horizon (7) subject to bioeconomic dynamic system (1) is defined as

$$
\begin{aligned}
H(x, k, \beta, \lambda, c, d) & =\eta \ln x+\nu \ln c \\
& +\beta\left[r x\left(1-\frac{x}{K}\right)-\epsilon k^{\alpha}+\sigma d^{\mu}\right]+\lambda\left[k^{\alpha}-c-d\right] .
\end{aligned}
$$

Here $\beta$ and $\lambda$ are adjoint variables (or co-states) associated with $x$ and $k$, respectively, and satisfy the following adjoint equations:

$$
\left\{\begin{array}{l}
\frac{d \beta}{d t}=\rho \beta-H_{x}(x, k, \beta, \lambda, c, d)=(\rho-r) \beta+\frac{2 \beta r}{K} x-\frac{\eta}{x} \\
\frac{d \lambda}{d t}=\rho \lambda-H_{k}(x, k, \beta, \lambda, c, d)=\left(\rho-\alpha k^{\alpha-1}\right) \lambda+\epsilon \alpha k^{\alpha-1} \beta
\end{array}\right.
$$

The transversality conditions ${ }^{2}$ are given by

$$
\lim _{T \rightarrow \infty} e^{-\rho T} x(T) \beta(T)=0, \quad \lim _{T \rightarrow \infty} e^{-\rho T} k(T) \lambda(T)=0 .
$$

Applying the maximum principle of L. S. Pontryagin (see e.g., [5]), we obtain the first-order necessary conditions of optimality:

$$
\frac{\partial H}{\partial c}=\frac{\nu}{c}-\lambda=0, \quad \frac{\partial H}{\partial d}=\sigma \mu d^{\mu-1} \beta-\lambda=0
$$

and observe that

$$
D_{(c, d)}^{2} H=\left[\begin{array}{ll}
H_{c c} & H_{c d} \\
H_{d c} & H_{d d}
\end{array}\right]=\left[\begin{array}{cc}
-\frac{\nu}{c^{2}} & 0 \\
0 & \sigma \mu(\mu-1) d^{\mu-2} \beta
\end{array}\right]
$$

\footnotetext{
${ }^{2}$ It is usually assumed that the transversality conditions in "standard" form (11) hold automatically, at least when $\rho>0$. However, this is not always true since the adjoint variables may exhibit various kinds of "pathological" asymptotic behavior at infinity. For more details regarding the behavior of adjoint variable at infinity and formal proofs of different types of transversality conditions the works [3] (and references therein) will be particularly useful.
} 
where $H_{c c}<0$ and $\operatorname{det} D_{(c, d)}^{2} H>0$ as long as $\beta>0$ (note that $\mu \in(0,1)$ and, therefore, $\left.H_{d d}<0\right)$. Thus, the Hamiltonian (9) attains its maximum when

$$
c^{*}=\frac{\nu}{\lambda}, \quad d^{*}=\left(\frac{\sigma \mu \beta}{\lambda}\right)^{\frac{1}{1-\mu}} .
$$

Additionally, it should be fulfilled that $\beta, \lambda>0$ in order to guarantee that $c^{*}, d^{*}>0$. If $\beta, \lambda>0$ for all $t>0$, the Mangasarian and Arrow sufficiency conditions for infinite-time horizon ${ }^{3}$ may also be fulfilled under some additional assumptions.

The substitution the optimal choices $c^{*}$ and $d^{*}$ from (13) in two dynamic systems $(1)$ and $(10)$ will result in $(4 \times 4)$ optimality system with respect to the variables $(x, k, \beta, \lambda)$ :

$$
\begin{aligned}
\frac{d x}{d t} & =r x\left(1-\frac{x}{K}\right)-\epsilon k^{\alpha}+\sigma\left(\frac{\sigma \mu \beta}{\lambda}\right)^{\frac{\mu}{1-\mu}} \\
\frac{d k}{d t} & =k^{\alpha}-\frac{\nu}{\lambda}-\left(\frac{\sigma \mu \beta}{\lambda}\right)^{\frac{1}{1-\mu}} \\
\frac{d \beta}{d t} & =\left(r\left(\frac{2 x}{K}-1\right)+\rho\right) \beta-\frac{\eta}{x} \\
\frac{d \lambda}{d t} & =\alpha(\epsilon \beta-\lambda) k^{\alpha-1}+\rho \lambda
\end{aligned}
$$

with initial conditions $x\left(t_{0}\right)=x_{0}, k\left(t_{0}\right)=k_{0}$ and the transversality conditions (11). Solution of this ODE system is quite a challenge, since there is no known technique for solving it analytically and for numerical solution the initial conditions $\beta\left(t_{0}\right)$ and $\lambda\left(t_{0}\right)$ are not provided.

However, it is clear that the transversality conditions (11) will be always satisfied by any trajectory approaching some fixed point $\left(x^{*}, k^{*}, \beta^{*}, \lambda^{*}\right)$ of the system (14). Therefore, by choosing $\beta\left(t_{0}\right)=\beta^{*}$ and $\lambda\left(t_{0}\right)=\lambda^{*}$ we can manage to satisfy the transversality conditions (11) and obtain fair initial guesses for numerical integration of the system (14). The latter has no contradictions with theoretical analysis rigourously performed in [3].

By analyzing the dynamics of the optimality system, it can be shown that stability properties of bioeconomic system (1) will be preserved under the optimal choice (13). Namely, for an initial state $\left(x_{0}, k_{0}\right)$ sufficiently close the fixed-point values $\left(x^{*}, k^{*}\right)$ it is possible to determine the initial values of multipliers $\left(\beta\left(t_{0}\right), \lambda\left(t_{0}\right)\right)$ such that the optimal economic growth trajectory could

\footnotetext{
${ }^{3} \mathrm{~A}$ formal statement of the Mangasarian and Arrow sufficiency conditions can be consulted in $[9, \mathrm{p} .159]$
} 
eventually converge to the fixed point of the optimality system (14) under the optimal choice of controls (13). On the other hand, such fixed point will be attainable by means of optimal strategies (13) only if the Jacobian matrix of the optimality system, evaluated in the fixed point, admits two or more eigenvalues with strictly negative real part.

\section{Existence of Fixed Points of the Optimality System}

Fixed points of the optimality system (14) are solutions of the following nonlinear algebraic system:

$$
\begin{aligned}
r x\left(1-\frac{x}{K}\right)-\epsilon k^{\alpha}+\sigma\left(\frac{\sigma \mu \beta}{\lambda}\right)^{\frac{\mu}{1-\mu}} & =0 \\
k^{\alpha}-\frac{\nu}{\lambda}-\left(\frac{\sigma \mu \beta}{\lambda}\right)^{\frac{1}{1-\mu}} & =0 \\
\left(r\left(\frac{2 x}{K}-1\right)+\rho\right) \beta-\frac{\eta}{x} & =0 \\
\alpha(\epsilon \beta-\lambda) k^{\alpha-1}+\rho \lambda & =0
\end{aligned}
$$

For positive $\beta$ and $\lambda$, equation (15d) makes sense only if the following relation

$$
\epsilon \beta-\lambda<0
$$

holds for $\beta, \lambda, \epsilon>0$. This is a necessary condition of solvability of the system (15) which was introduced and fully justified in [6]. On the other hand, equation (15c) is quadratic in $x$, that is,

$$
\frac{2 r}{K} x^{2}-(r-\rho) x-\frac{\eta}{\beta}=0,
$$

and yields two different real values for $x$, namely,

$$
x_{ \pm}=\frac{K}{4 r}\left(r-\rho \pm \sqrt{(r-\rho)^{2}+\frac{8 r \eta}{\beta K}}\right) .
$$

However, it is worthwhile to note that $x_{-}<0$ and we end up with only one positive root, $x_{+}>0$, given that $\beta>0$. On the other hand, this root is feasible in the the sense that $0 \leq x_{+} \leq K$, only if

$$
-\rho+\sqrt{(r-\rho)^{2}+\frac{8 r \eta}{\beta K}} \leq 3 r \quad \Rightarrow \quad \beta \geq \frac{\eta}{K(r+\rho)}=\hat{\beta} .
$$




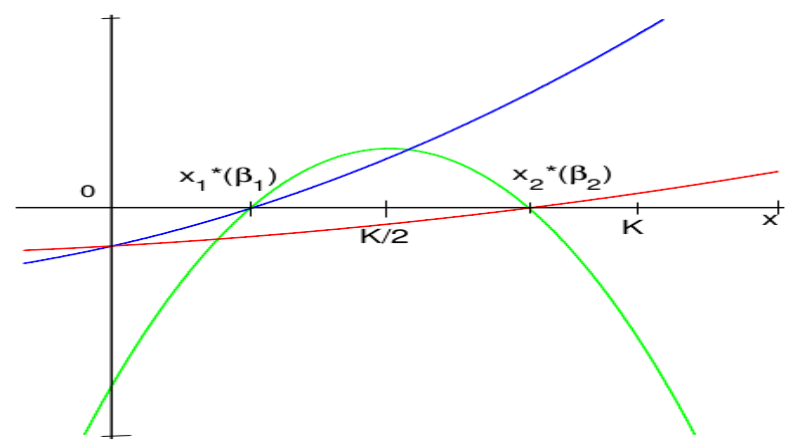

Figure 2: Graphic solution of the system (15): case with $\beta_{1}>\beta_{2}>\hat{\beta}$ and $0<x_{1}\left(\beta_{1}\right)<\frac{K}{2}<x_{2}\left(\beta_{2}\right)<K$.

Here the lower bound, $\hat{\beta}$, correspond to "pure" logistic case since for $\beta=\hat{\beta}$ we have $x_{1}=K$. Moreover, the vertex $\hat{x}$ of the parabola (17) always lays below the $x$-axis and

$$
\hat{x}=\frac{r-\rho}{4 r} K= \begin{cases}<0, & \text { if } \quad r<\rho \\ =0, & \text { if } \quad r=\rho \\ >0, & \text { if } \quad r>\rho\end{cases}
$$

Given that in our case $r<\rho$, the vertex $\hat{x}$ lays in the left lower quadrant for any positive value of $\beta$. This parabola has variable width defined by the value of $\beta$ (see Eq. (17)) that solves other equations of the system (15).

Figure 2 displays the relationship between two feasible symmetric solutions $\left(0<x_{1}<\frac{K}{2}<x_{2}<K, x_{1}+x_{2}=K\right)$ of perturbed logistic equation (15a) and positive root $x_{+}$of quadratic equation (17) for two different values of $\beta$. It is worthwhile to note that parabola (17) always has negative intersection with the vertical axis for any positive $\beta$. Additionally, when $\beta \rightarrow \hat{\beta}$ we have $x_{2} \rightarrow K$, while $\beta \rightarrow \infty$ implies that $x_{1} \rightarrow 0$ ("pure" logistic case).

For the solvability analysis of (15), let us fix all constant parameters of the model except $\epsilon>0$ and then try to parameterize the solution $\left(x^{*}, k^{*}, \beta^{*}, \lambda^{*}\right)$ of (15) with $\epsilon$. First we express our state variables $x$ and $k$ in terms of the adjoint variables $\beta$ and $\lambda$ using equations (15b), (15c):

$$
x=\frac{K}{4 r}\left(r-\rho+\sqrt{(r-\rho)^{2}+\frac{8 r \eta}{\beta K}}\right), \quad k^{\alpha}=\frac{\nu}{\lambda}+\left(\frac{\sigma \mu \beta}{\lambda}\right)^{\frac{1}{1-\mu}}
$$

and then substitute them into (15a) and (15d), in order to obtain a reduced 
system of two equations in the variables $\beta$ and $\lambda$ :

$$
\begin{aligned}
F_{1}(\beta, \lambda) & \triangleq \frac{K}{4}\left[r-\rho+\sqrt{(r-\rho)^{2}+\frac{8 r \eta}{\beta K}}\right] \\
& -\frac{K}{16 r}\left[r-\rho+\sqrt{(r-\rho)^{2}+\frac{8 r \eta}{\beta K}}\right]^{2} \\
& -\epsilon\left[\frac{\nu}{\lambda}+\left(\frac{\sigma \mu \beta}{\lambda}\right)^{\frac{1}{1-\mu}}\right]+\sigma\left(\frac{\sigma \mu \beta}{\lambda}\right)^{\frac{\mu}{1-\mu}}=0, \\
F_{2}(\beta, \lambda) & \triangleq \alpha(\beta \epsilon-\lambda)\left[\frac{\nu}{\lambda}+\left(\frac{\sigma \mu \beta}{\lambda}\right)^{\frac{1}{1-\mu}}\right]^{\frac{\alpha-1}{\alpha}}+\rho \lambda=0 .
\end{aligned}
$$

Thus we arrive to a system of two nonlinear equations with two variables $(\beta, \lambda)$ depending on parameter $\epsilon$ which has been left free. If (20)-(21) is solvable in terms of $\beta^{*}=\beta(\epsilon), \lambda^{*}=\lambda(\epsilon)$ with some $\epsilon>0$, then the original system (15) will be also solvable for $x^{*}=x(\epsilon), k^{*}=k(\epsilon)$ by means of relationships (19).

The existence of $\epsilon$-dependent solution $(\beta(\epsilon), \lambda(\epsilon))$ of the system $(20)-(21)$ can be established on the basis of the Implicit Function Theorem.

Theorem 2. There exists a unique reference value $\epsilon_{*}>0$ of parameter $\epsilon$ (solely defined by the model's parameters $r, K, \alpha, \mu, \sigma)$ such that

$$
\begin{gathered}
x_{1}^{*}\left(\epsilon_{*}\right)=\frac{K}{4}, \quad x_{2}^{*}\left(\epsilon_{*}\right)=\frac{3 K}{4}, \quad x_{1}^{*}\left(\epsilon_{*}\right)+x_{2}^{*}\left(\epsilon_{*}\right)=K, \\
\beta_{1}^{*}\left(\epsilon_{*}\right)=\frac{8 \eta}{K(2 \rho-r)}, \quad \beta_{2}^{*}\left(\epsilon_{*}\right)=\frac{8 \eta}{3 K(2 \rho+r)}, \quad \beta_{1}^{*}\left(\epsilon_{*}\right)>\beta_{2}^{*}\left(\epsilon_{*}\right), \\
\lambda_{i}^{*}\left(\epsilon_{*}\right)=2 \epsilon_{*} \beta_{i}\left(\epsilon_{*}\right), \quad k_{i}^{*}\left(\epsilon_{*}\right)=\left[\frac{\nu}{\lambda_{i}\left(\epsilon_{*}\right)}+\left(\frac{\sigma \mu \beta_{i}\left(\epsilon_{*}\right)}{\lambda_{i}\left(\epsilon_{*}\right)}\right)^{\frac{1}{1-\mu}}\right]^{\frac{1}{\alpha}}, \quad i=1,2
\end{gathered}
$$

Additionally, $\beta_{i}\left(\epsilon_{*}\right)>0, \lambda_{i}\left(\epsilon_{*}\right)>0$ and they comply with (16) for $i=1,2$. Therefore, the subsystem (20)-(21) has $\epsilon$-dependent solution extendible to the open vicinities $\mathcal{V}_{i}=I_{1}\left(\beta_{i}\right) \times I_{2}\left(\lambda_{i}\right) \times I_{3}(\epsilon) \subset \mathbb{R}_{+}^{3}$ of $\left(\beta_{i}\left(\epsilon_{*}\right), \lambda_{i}\left(\epsilon_{*}\right) ; \epsilon_{*}\right), i=1,2$ in the sense that

$$
F_{1}(\beta(\epsilon), \lambda(\epsilon) ; \epsilon)=0, \quad F_{2}(\beta(\epsilon), \lambda(\epsilon) ; \epsilon)=0
$$

Proof. Using four equations (15), we need to solve the reduced system (20)(21) in the variables $\beta=\beta(\epsilon)$ and $\lambda=\lambda(\epsilon)$, where it is required that (16) holds. 
Using the equation (21) in (20) we can further reduce the system system (20)-(21) to a single equation:

$$
\begin{aligned}
F(\beta, \lambda ; \epsilon) \triangleq \frac{K}{4}[r-\rho+ & \left.\sqrt{(r-\rho)^{2}+\frac{8 r \eta}{\beta K}}\right] \\
-\frac{K}{16 r} & {\left[r-\rho+\sqrt{(r-\rho)^{2}+\frac{8 r \eta}{\beta K}}\right]^{2} } \\
& -\epsilon\left[\frac{\alpha}{\rho}\left(1-\frac{\epsilon \beta}{\lambda}\right)\right]^{\frac{\alpha}{1-\alpha}}+\sigma\left(\frac{\sigma \mu \beta}{\lambda}\right)^{\frac{\mu}{1-\mu}}=0 .
\end{aligned}
$$

In order to eliminate both radicals, we set $\beta=\beta_{i}^{*}$ and $\lambda_{i}^{*}=2 \epsilon_{*} \beta_{i}^{*}, i=1,2$ for both values of $\beta_{i}^{*}$ and $\lambda_{i}^{*}$ given in (23) and (24), respectively. In both cases we arrive to

$$
\begin{aligned}
F\left(\beta_{i}^{*}, \lambda_{i}^{*} ; \epsilon_{*}\right) & =\frac{3 K r}{16}-\epsilon_{*}\left[\frac{\alpha}{\rho}\left(1-\frac{\epsilon_{*} \beta_{i}^{*}}{\lambda_{i}^{*}}\right)\right]^{\frac{\alpha}{1-\alpha}}+\sigma\left(\frac{\sigma \mu \beta_{i}^{*}}{\lambda_{i}^{*}}\right)^{\frac{\mu}{1-\mu}} \\
& =\frac{3 K r}{16}-\epsilon_{*}\left[\frac{\alpha}{2 \rho}\right]^{\frac{\alpha}{1-\alpha}}+\sigma\left[\frac{\sigma \mu}{2 \epsilon_{*}}\right]^{\frac{\mu}{1-\mu}}=0
\end{aligned}
$$

In other words, $\epsilon_{*}$ must be a root of nonlinear algebraic equation $S(\epsilon)=0$ where real function $S:(0, \infty) \rightarrow \mathbb{R}$ is defined by

$$
S(\epsilon)=\frac{3 K r}{16}-\epsilon\left[\frac{\alpha}{2 \rho}\right]^{\frac{\alpha}{1-\alpha}}+\sigma\left[\frac{\sigma \mu}{2 \epsilon}\right]^{\frac{\mu}{1-\mu}} .
$$

Function $S(\epsilon)$ is continuous for $\epsilon \in(0, \infty)$ and it is also true that

$$
\lim _{\epsilon \rightarrow \infty} S(\epsilon)=-\infty, \quad \lim _{\epsilon \rightarrow 0^{+}} S(\epsilon)=+\infty
$$

Additionally, $S^{\prime}(\epsilon)<0$ for all $\epsilon>0$ so that $S(\epsilon)$ is strictly decreasing and changes its sign only once within $(0, \infty)$. In virtue of Bolzano's theorem, there must exist exactly one $\epsilon_{*}>0$ such that $S\left(\epsilon_{*}\right)=0$.

Thus, it has been proved that there exists a unique reference value $\epsilon_{*}>0$ solely defined by the model's parameters as a root of function $S(\epsilon)$ (see Eq. (26)) for which $F(\beta, \lambda ; \epsilon)=0$ given by (25) is solvable.

It is also clear that

$$
\frac{\partial F}{\partial \lambda}=-\frac{\beta}{\lambda^{2}}\left[\frac{\alpha \epsilon^{2}}{1-\alpha}\left(\frac{\alpha}{\rho}\right)^{\frac{\alpha}{1-\alpha}}\left(1-\frac{\epsilon \beta}{\lambda}\right)^{\frac{2 \alpha-1}{1-\alpha}}+\frac{\sigma^{2} \mu^{2}}{1-\mu}\left(\frac{\sigma \mu \beta}{\lambda}\right)^{\frac{2 \mu-1}{1-\mu}}\right]<0
$$


for all positive $\beta, \lambda, \epsilon$ satisfying (16).

Consequently, $F\left(\beta_{i}^{*}, \lambda_{i}^{*}, \epsilon_{*}\right)=0$ and $\frac{\partial}{\partial \lambda} F\left(\beta_{i}^{*}, \lambda_{i}^{*}, \epsilon_{*}\right) \neq 0$. According to Implicit Function Theorem, there must exist open vicinities $\mathcal{V}_{i}=I_{1}\left(\beta_{i}\right) \times$ $I_{2}\left(\lambda_{i}\right) \times I_{3}(\epsilon) \subset \mathbb{R}_{+}^{3}$ of $\left(\beta_{i}\left(\epsilon_{*}\right), \lambda_{i}\left(\epsilon_{*}\right), \epsilon_{*}\right), i=1,2$ and differentiable functions $\lambda_{i}: I_{1}\left(\beta_{i}\right) \times I_{3}(\epsilon) \rightarrow I_{2}\left(\lambda_{i}\right)$ such that for any $\left(\beta_{i}, \epsilon\right) \in I_{1}\left(\beta_{i}\right) \times I_{3}(\epsilon)$ it holds that $F\left(\beta_{i}, \lambda_{i}\left(\beta_{i}, \epsilon\right), \epsilon\right)=0, i=1,2$. Additionally, since both $\beta_{i}$ depend implicitly on $\epsilon$, we actually have

$$
F\left(\beta_{i}(\epsilon), \lambda_{i}\left(\beta_{i}(\epsilon), \epsilon\right), \epsilon\right)=0, \quad i=1,2 .
$$

Using the form of $F$ given by (25), this last equation can be split backwards (for each $i=1,2$ ) in two equations of the form

$$
F_{1}\left(\beta_{i}(\epsilon), \lambda_{i}(\epsilon), \epsilon\right)=0, \quad F_{2}\left(\beta_{i}(\epsilon), \lambda_{i}(\epsilon), \epsilon\right)=0
$$

where $\lambda_{i}(\epsilon)=\lambda_{i}\left(\beta_{i}(\epsilon), \epsilon\right)$ is an implicit solution of (21) for $\epsilon \in I_{3}(\epsilon)$ under the condition (16) and $F_{1}, F_{2}$ are given by (20) and (21), respectively.

Using the one-to-one correspondence between $\beta$ and $x$ as well as explicit formulas for $k$ (see relationships in (19)), it is easy to calculate that $x_{1}^{*}=$ $K / 4, x_{2}^{*}=3 K / 4$ (cf. (22)) and $k_{i}^{*}, i=1,2$ evaluated for corresponding $\beta_{i}^{*}, \lambda_{i}^{*}$ (cf. (24)) are solutions of the original fixed-point system (15) when $\epsilon=\epsilon_{*}$. This proves all statements of the theorem.

Corollary 3. For all $\epsilon \in I_{3}(\epsilon)$ defined by the Theorem 2, the following parametric curves

$$
\begin{array}{ll}
\beta_{1}(\epsilon), \beta_{2}(\epsilon): & I_{3} \rightarrow(\hat{\beta}, \infty) \subset \mathbb{R}_{+} \\
\lambda_{1}(\epsilon), \lambda_{2}(\epsilon): & I_{3} \rightarrow \mathbb{R}_{+} \\
x_{1}(\epsilon), x_{2}(\epsilon): & I_{3} \rightarrow[0, K] \subset \mathbb{R}_{+} \\
k_{1}(\epsilon), k_{2}(\epsilon): & I_{3} \rightarrow \mathbb{R}_{+}
\end{array}
$$

satisfy the system (15) and thus provide two feasible fixed points of optimality system (14) for corresponding value of $\epsilon$ :

$$
P_{1}^{*}=\left(x_{1}(\epsilon), k_{1}(\epsilon), \beta_{1}(\epsilon), \lambda_{1}(\epsilon)\right) \quad \text { and } \quad P_{2}^{*}=\left(x_{2}(\epsilon), k_{2}(\epsilon), \beta_{2}(\epsilon), \lambda_{2}(\epsilon)\right) .
$$

Additionally, it is fulfilled that

$$
x_{1}(\epsilon)+x_{2}(\epsilon)=K, \quad \hat{\beta}=\frac{\eta}{K(\rho+r)}, \quad \epsilon \beta_{i}(\epsilon)-\lambda_{i}(\epsilon)<0, \quad i=1,2
$$




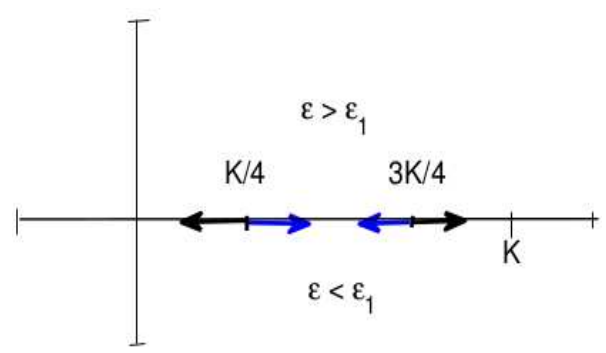

Figure 3: Displacements of $x_{1}(\epsilon)$ and $x_{2}(\epsilon)$ caused by alterations in $\epsilon$.

$$
\sqrt{(r-\rho)^{2}+\frac{8 r \eta}{\beta_{1}^{*} K}}+\sqrt{(r-\rho)^{2}+\frac{8 r \eta}{\beta_{2}^{*} K}}=2 \rho+2 r .
$$

Effectively, any alteration of $\epsilon$ from its reference value $\epsilon_{*}$ would cause $x_{1}(\epsilon)$ and $x_{2}(\epsilon)$ to move either towards the center $K / 2$ of $[0, K]\left(\epsilon>\epsilon_{*}\right)$ or towards its end-points $\left(\epsilon<\epsilon_{*}\right)$; see Figure 3. At any case, due to the symmetry with respect to $K / 2$ and in view of the first relationship in (19), the corresponding $\beta_{1}(\epsilon)$ and $\beta_{2}(\epsilon)$ must satisfy the condition (27).

It is worthwhile to note that if $x_{1}(\epsilon)=x_{2}(\epsilon)=K / 2$ (for some $\epsilon$ ) it should be also fulfilled that

$$
\beta_{1}(\epsilon)=\beta_{2}(\epsilon)=\frac{2 \eta}{\rho K}
$$

for the same $\epsilon$.

\section{Practical Applications and Discussion}

Existence of two fixed points $P_{1}^{*}$ and $P_{2}^{*}$ of the system (15) has been proved in the previous section; however, their calculation can be only carried out by some numerical procedure (e.g. Newton-type methods or other techniques). Therefore, we should need some "reasonable guess" for a target point $P_{2}^{*}$ (where $x$-component has maximal species abundance).

The "reference value" of $\epsilon$ (i.e., $\epsilon_{*}$ defined as a root of function $S(\epsilon)$ given by (26)) can be calculated using the constant parameters of the model. This value $\epsilon_{*}$ will indicate the position of $x_{2}(\epsilon)$-component (for $\epsilon$ actually given in the model) with respect to its "reference position" $x_{2}^{*}\left(\epsilon_{*}\right)=3 K / 4$ (that is, on the left of $3 K / 4$ if $\epsilon>\epsilon_{*}$ or on the right of $3 K / 4$ if $\epsilon<\epsilon_{*}$ - see Figure 3). 
Thus, we may use the following set of "reasonable initial guesses":

$$
\tilde{x}=\frac{3 K}{4}, \tilde{\beta}=\frac{8 \eta}{3 K(2 \rho+r)}, \tilde{\lambda}=2 \epsilon \tilde{\beta}, \tilde{k}=\left[\frac{\nu}{\tilde{\lambda}}+\left(\frac{\sigma \mu \tilde{\beta}}{\tilde{\lambda}}\right)^{\frac{1}{1-\mu}}\right]
$$

with $\epsilon, \sigma, r, \rho, K, \nu, \alpha$, and $\mu$ given in the model.

Remark 4. To be on a safe side, we may also try two alternative sets of initial guesses:

$$
\hat{x}=K, \quad \hat{\beta}=\frac{\eta}{K(\rho+r)}, \quad \hat{\lambda}=2 \epsilon \hat{\beta}, \quad \hat{k}=\left[\frac{\nu}{\hat{\lambda}}+\left(\frac{\sigma \mu \hat{\beta}}{\hat{\lambda}}\right)^{\frac{1}{1-\mu}}\right]^{\frac{1}{\alpha}}
$$

when $\epsilon<\epsilon_{*}$ or

$$
\check{x}=\frac{K}{2}, \quad \check{\beta}=\frac{2 \eta}{\rho K}, \quad \check{\lambda}=2 \epsilon \check{\beta}, \quad \check{k}=\left[\frac{\nu}{\check{\lambda}}+\left(\frac{\sigma \mu \check{\beta}}{\check{\lambda}}\right)^{\frac{1}{1-\mu}}\right]^{\frac{1}{\alpha}}
$$

when $\epsilon>\epsilon_{*}$ and thus to crosscheck the result obtained with initial guess (28).

Once all the components of $P_{2}^{*}=\left(x^{*}, k^{*}, \beta^{*}, \lambda^{*}\right)$ are defined, our goal is to move the trajectories $(x(t), k(t), \beta(t), \lambda(t))$ of the optimality system (14) towards the fixed point $P_{2}^{*}=\left(x^{*}, k^{*}, \beta^{*}, \lambda^{*}\right)$ with maximal species abundance at indefinite time (that is, when $t \rightarrow \infty)$. According to [8], a generic $(4 \times 4)$ optimality system has a saddle point which is reachable (in infinite time) if the initial values of the state variables $\left(x(0)=x_{0}\right.$ and $k(0)=k_{0}$, in our case) are situated closely enough to its saddle-point values.

In such a case, we should set the optimal choices for control variables $c^{*}$ and $d^{*}$ according to (13) by using the numerical values of $\beta^{*}, \lambda^{*}$, that is:

$$
c^{*}=\frac{\nu}{\lambda^{*}}, \quad d^{*}=\left(\frac{\sigma \mu \beta^{*}}{\lambda^{*}}\right)^{\frac{1}{1-\mu}} .
$$

If $x(0)=x_{0}>K / 2$ (that is, $x_{0}$ is located closer to $3 K / 4$ than to $K / 4$ ), then under this policy, we can guarantee a sustainable conservation of the species.

For practical calculations, the transversality conditions (11) should be replaced by initial conditions

$$
\beta(0)=\beta^{*}, \quad \lambda(0)=\lambda^{*}
$$




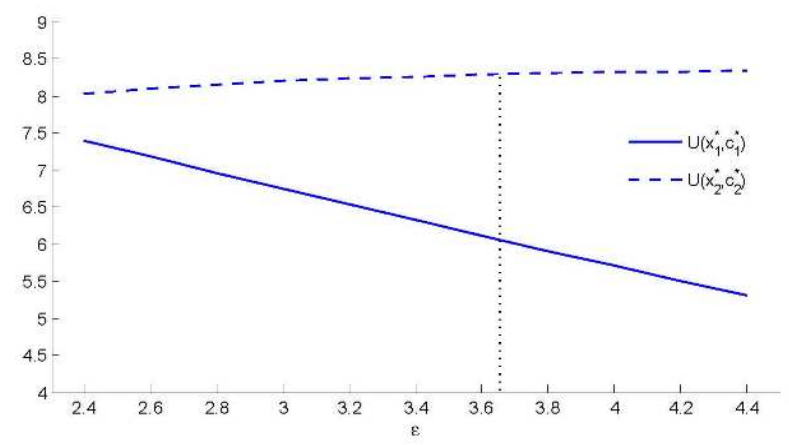

Figure 4: Fixed-point utilities $U\left(x_{1}^{*}, c_{1}^{*}\right)$ and $U\left(x_{2}^{*}, c_{2}^{*}\right)$ as functions of $\epsilon$.

and the optimality system (14) should be integrated numerically forward in time using the set of four initial conditions:

$$
x(0)=x_{0}, \quad k(0)=k_{0}, \quad \beta(0)=\beta^{*}, \quad \lambda(0)=\lambda^{*}
$$

from $t=0$ to $t=\infty$ (in theory) or to $t=T$ where $T \gg 0$ is sufficiently large (in practice). Further, the overall discounted utility can be estimated by evaluating the integral (7).

Figure 4 displays the $\epsilon$-dependent utility function $U(x(\epsilon), c(\epsilon))$ evaluated in both fixed point for different values of $\epsilon$. The vertical dotted line marks the reference value $\epsilon_{*} \approx 3.6491$ (i.e., root of function $S(\epsilon)$ - see formula $(26)$ ) calculated for numerical values of other parameters borrowed from [6], namely:

$$
r=0.05, K=500, \sigma=10, \mu=0.7, \alpha=\frac{2}{3}, \rho=0.1398, \eta=1, \nu=202 .
$$

From the Figure 4 it becomes clear that the fixed point with maximal species abundance $\left(P_{2}^{*}=\left(x^{*}, k^{*}, \beta^{*}, \lambda^{*}\right)\right)$ always guarantees greater utility.

\section{Conclusions}

The controversy between the preservation of biological species and the inevitable spread of anthropic activities has been on debated among many scholars debate during last decades. In effect, rather common wildlife species in South America (such as armadillo, capybara, wild boar, etc.) are becoming scarce and even locally extinct not only around of megalopolis zones but also within national parks and other reservation areas. The latter occurs not because of 
animal slaughter but because of lack of food, water or land resources necessary for the species survival and reproduction. To counterbalance this tendency, policy makers should be compelled to implement defensive actions aimed at sustainable conservation of biological species from local extinction.

The results obtained in this paper suggest the existence of sustainable decision policies capable to to maintain the wildlife stock at some reasonable level and thus to avoid local extinction of single biological species.

\section{Acknowledgments}

This research was supported by Universidad del Valle (Cali, Colombia) by way of the Research Project CI-7939 "Optimization and Viability Models in Ecology and Economics - ECOPTIM" endorsed by PEERS-IRD, France within the frameworks of REALMA Network (Réseau Europe - Amérique Latine en Mathématiques Appliquées, http://www.realma.org/).

\section{References}

[1] Robert R. Alexander and David W. Shields. Using land as a control variable in density-dependent bioeconomic models. Ecological Modelling, 170(2-3):193-201, 2003.

[2] Angelo Antoci, Simone Borghesi, and Paolo Russu. Biodiversity and economic growth: Trade-offs between stabilization of the ecological system and preservation of natural dynamics. Ecological Modelling, 189(3-4):333$346,2005$.

[3] S. Aseev and A. Kryazhimskii. The pontryagin maximum principle and optimal economic growth problems. Proceedings of the Steklov Institute of Mathematics, 257:1-255, 2007.

[4] Doris E. Campo-Duarte and Olga Vasilieva. Bioeconomic model with Gompertz population growth and species conservation. Int. J. Pure Appl. Math., 72(1):49-63, 2011.

[5] Colin W. Clark. Mathematical bioeconomics: the optimal management of renewable resources. Wiley-Interscience [John Wiley \& Sons], New York, 1976. 
[6] Erica Cruz-Rivera and Olga Vasilieva. Optimal policies aimed at stabilization of populations with logistic growth under human intervention. Theoretical Population Biology, 83:123-135, 2013.

[7] Erica Cruz-Rivera, Olga Vasilieva, and Mikhail Svinin. Optimal short-term policies for protection of single biological species from local extinction. Ecological Modelling, 263:273-280, 2013.

[8] Engelbert Dockner. Local stability analysis in optimal control problems with two state variables. In Optimal control theory and economic analysis, 2 (Vienna, 1984), pages 89-103. North-Holland, Amsterdam.

[9] Dieter Grass, Jonathan P. Caulkins, Gustav Feichtinger, Gernot Tragler, and Doris A. Behrens. Optimal control of nonlinear processes with applications in drugs, corruption, and terror. Springer-Verlag, Berlin, 2008.

[10] Olga Vasilieva. From harvesting to non-harvesting utility: an optimal control approach to species conservation. Natural Resource Modeling, 2015. forthcoming. 
